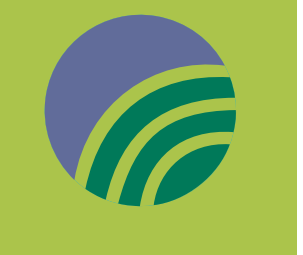

\section{Robert Cammarata of Johns Hopkins University remembered}

$\mathrm{R}$ obert "Bob" Cammarata, professor of materials science and engineering at Johns Hopkins University, died on Wednesday, January 13, 2016, at the age of 58. Cammarata served as chair of the Department of Materials Science and Engineering at Johns Hopkins from 2003 until 2008. He had been a member of the Johns Hopkins Materials Research, Science, and Engineering Center and, more recently, the Hopkins Extreme Materials Institute. He was affiliated with the Department of Mechanical Engineering, as well.

Bob was "an insightful scientist, a talented teacher, a visionary entrepreneur, a mentor to students and colleagues, and a leader in the Johns Hopkins community," said T.E. "Ed" Schlesinger, Dean of the Johns Hopkins Whiting School of Engineering.

Bob joined the Johns Hopkins Whiting School faculty in 1987 after completing a bachelor's degree in materials science from the Massachusetts Institute of Technology (MIT), a doctorate in applied physics at Harvard University, and subsequent postdoctoral research at MIT and IBM. At Johns Hopkins, he oversaw a vibrant research program for which he received significant external recognition, especially for his studies on the fundamental thermodynamics and mechanics of thin films.

Bob became a full professor and chair of the Materials Science and Engineering Department just when biotechnology and nanotechnology concentrations began to take shape. He helped integrate these specialties with the fundamental understanding of the structures, energetics, kinetics, and mechanical behavior associated with materials and interface behavior, in which he was a renowned world leader. He authored some of the most authoritative reviews in these areas.

He was elected a Fellow of the Materials Research Society (MRS) in 2011 and the American Physical Society in 2012 for his pioneering contributions to the understanding of thermodynamics and mechanics of surfaces, thin films, and nanomaterials, and to the synthesis, processing, and mechanical behavior of nanocomposites. He was very active within MRS and was on the editorial boards of the Journal of Materials Research and MRS Bulletin for several years.

Despite his deep scholarship and love for thermodynamics fundamentals, theory and modeling, and materials processing and mechanics, he was open to the wider applicability of his ideas. Most recently, building on a collaborative research project involving electrophoretic manipulation of nanowires, he founded the company NanoDirect with his $\mathrm{PhD}$ advisee, Stephen Farias, to commercialize efficient and cost-effective ways of separating semiconducting and metallic nanotube and nanowire objects.

"He was really great and funny and, as a teacher, he was very good at taking complex thoughts and making them accessible to students. It was a real talent of his. Some professors would not take questions from students who were not in their class, but that was not the case with Bob," said Farias. "You could ask him questions, and he spent many extra hours with students. He was that kind of teacher."

Besides academic contributions, Bob gave enormously to the general welfare of Johns Hopkins. He served as an elected member of the Homewood Academic Council from 2006 to 2011. He also served as an unofficial mentor to numerous undergraduate, graduate, and professional members of the university community. In 2010, he was recognized with the McDonald Award for Excellence in Mentoring and Advising, a student-driven award given as a tribute to the insight and inspiration that Bob provided to all who were fortunate enough to find themselves in his classes or research circles.

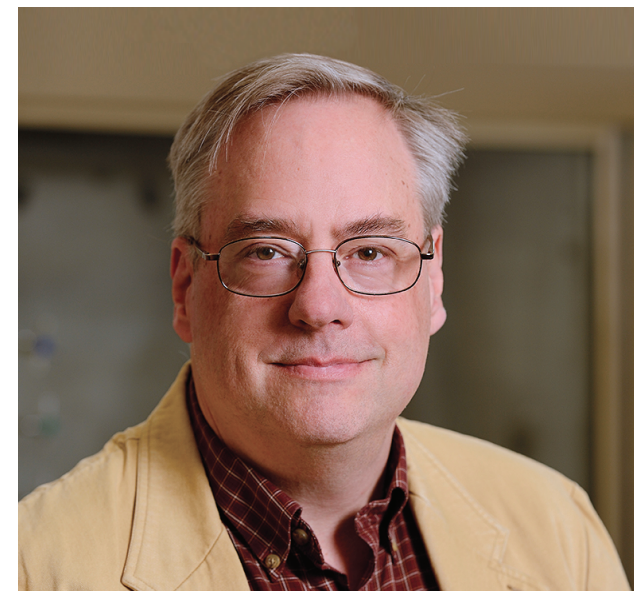

Bob also had numerous skills and interests outside of science. He was a master chess player who could win an online game while walking through thermodynamics equations. He was an expert fantasy (American) football player (and a really fun or insufferable fan, depending on one's allegiances relative to his), a drummer, a dedicated dog rescuer, and an outstanding comedian.

"I remember Bob as an unusual student, a valued colleague, and a great friend," said Frans Spaepen, Harvard School of Engineering and Applied Sciences. "His insistence on rigorous understanding of the fundamentals is an example to us all. His work on surface and interface stresses stimulated the application of these important concepts in new areas such as gels and other soft materials. His erudition and remarkable way with words made him a much beloved speaker and teacher, and a great spokesman for our field."

Numerous participants in a memorial program held for Bob at Johns Hopkins on March 13, 2016, remembered Bob as exceptionally fair, humble, caring, and generous to a fault, all with a lightheartedness that could brighten the most solemn occasions. This lightheartedness pervaded the memorial event. Too bad that it could not have come from Bob's own voice. Bob is survived by his wife, Sharka Prokes, his brother, Ronald Cammarata, and his sisterin-law, Norma Cammarata, to whom we offer our deepest condolences.

Howard Katz

Professor of Materials Science and Engineering at Johns Hopkins University and a colleague of Bob Cammarata. 